\title{
Overexpression of fibrinogen-like protein 2 alleviates acute rejection in rat models of liver transplantation
}

\author{
Xinxue Zhang", Jun Ma", Han Li, Lin Zhou, Zhe Liu, Shaocheng Lyu, Qiang He, Xianliang Li \\ Department of Hepatobiliary and Pancreaticosplenic Surgery, Beijing Chaoyang Hospital, Capital Medical University, Beijing, China \\ Contributions: (I) Conception and design: All authors; (II) Administrative support: X Li, Q He; (III) Provision of study materials or patients: X Li, \\ Q He; (IV) Collection and assembly of data: X Zhang, J Ma; (V) Data analysis and interpretation: All authors; (VI) Manuscript writing: All authors; \\ (VII) Final approval of manuscript: All authors. \\ "These authors contributed equally to this work. \\ Correspondence to: Qiang He; Xianliang Li. Department of Hepatobiliary and Pancreaticosplenic Surgery, Beijing Chaoyang Hospital, Capital Medical \\ University, 8 Gongtinan Road, Chaoyang District, Beijing 100020, China. Email: heqiang349@sina.com; dr_lxl@sina.com.
}

\begin{abstract}
Background: The role of cluster of differentiation (CD)8+ regulatory $T$ cells (Tregs) has previously been elucidated in tolerance models. Fibrinogen-like protein 2 (FGL2), that is secreted by Treg cells, which exhibited immunosuppressive functions, may alleviate acute rejection (AR). However, the precise role of CD8+ Tregs and FGL2 in the AR of rat liver transplantation remains unknown. Our previous study found that CD8+CD45RClow Tregs played crucial roles in maintaining immune tolerance. Here, we elucidated the role of CD8+ CD45RClow Treg and FGL2 in AR of rat liver transplantation.

Methods: A rat non-materialized AR of liver transplantation model was established using donors infected with no-load adeno-associated virus and adeno-associated virus expressing FGL2.

Results: There was an accumulation of tolerogenic CD8+CD45RClow in allografts compared with blank groups. Moreover, the proportion of CD8+CD45RClow Tregs was increased with longer survival time. Furthermore, we detected higher levels of FGL2 in the allografts infected with AAV-FGL2 in rats with AR of liver transplantation. We found that FGL2 could alleviate AR, and the survival time was prolonged in the recipients of donors infected with AAV-FGL2.

Conclusions: Our data suggest that CD8+CD45RClow Tregs was accumulated in allografts. The presence of FGL2 alleviated AR and prolonged survival time in the AR of liver transplantation rat model, suggesting that FGL2 and CD8+CD45RClow Tregs may serves as novel therapeutic targets for AR in liver transplantation.
\end{abstract}

Keywords: Fibrinogen-like protein 2 (FGL2); rejection; CD8 ${ }^{+}$CD45RC ${ }^{\text {low }}$ Treg; liver transplantation

Submitted Dec 08, 2020. Accepted for publication Feb 16, 2021.

doi: $10.21037 /$ atm-20-7881

View this article at: http://dx.doi.org/10.21037/atm-20-7881

\section{Introduction}

Liver transplantation is the only curative treatment for endstage liver disease and is considered one of the significant medical achievements of the last half-century (1). However, rejection is one of the main factors influencing the patients' outcome after liver transplantation and is a leading cause of death after transplant $(2,3)$. The crucial prevention and treatments of allograft rejection mainly depend on immunosuppressive therapy (4). However, the existing effective immunosuppressive agents are limited by their side effects, which include renal toxicity and neurotoxicity (5). Hence, more effective methods of preventing acute rejection (AR) need to be found.

Regulatory T cells (Tregs) have been most extensively studied in immune tolerance and are essential for transplantation tolerance (6-8). Several reports have indicated that Tregs accelerate apoptosis of $\mathrm{T}$ lymphocytes 
by depriving them of interleukin-2 (IL-2) or by releasing cytolytic molecules, such as granzyme B and perforin $(9,10)$. Effector lymphocytes can be inhibited directly via the secretion of suppressive cytokines, such as IL-10, IL-35, transforming growth factor- $\beta$ (TGF- $\beta$ ), and fibrinogen-like protein 2 (FGL2) (11,12).

An essential member of the fibrinogen-related protein superfamily, FGL2, includes both soluble and membrane subtypes, which contain a fibrinogen-related domain (FReD) $(13,14)$. The FReD region is critical to immunoregulatory activity. The FGL2 has been recognized as an inhibitory cytokine and plays a crucial role in innate and adaptive immunity (15). Secreted by Tregs, FGL2 binds to Fc $\gamma$ RIIB/ RIII expressed on antigen-presenting cells (APCs), including macrophages, dendritic cells (DC), and B cells (16). This action inhibits the maturation of APCs, which results in a reduction in $\mathrm{T}$ effector cell functions $(13,16-18)$. Reports have shown that soluble fibrinogen like protein 2 (sFGL2), an immune regulator, plays an essential role in the immune balance and in autoimmune diseases $(19,20)$, and an association has been found between FGL2 and allograft tolerance. Furthermore, inhibition of FGL2 reverses the tolerizing effects of rapamycin (14). A variety of studies have reported on FGL2 expression in Tregs $(15,21)$. Our report that FGL2 expression and cluster of differentiation (CD)8+CD45RClow Tregs were increased in tolerance groups of rat orthoptic liver transplant (OLT). The goal of this study was to further explore the role of CD8+CD45RClow Tregs and FGL2 in alleviation of rejection in a rat OLT model.

We present the following article following the ARRIVE reporting checklist (available at http://dx.doi.org/10.21037/ atm-20-7881).

\section{Methods}

\section{Ethical approval}

This study was performed under a project license (2019-D.102) granted by the Ethics Committee of Beijing Chaoyang Hospital and complied with the institutional guidelines for the care and use of animals.

\section{Animals and rat transplantation models}

Male Lewis and Brown Norwegian (BN) rats, aged 810 weeks old, were purchased from Beijing Vitonglihua Laboratory Animal Technology Co., Ltd. (Beijing, China).
All rats were maintained under specific pathogen-free conditions in the basic Medical Research Center of Beijing Chaoyang Hospital affiliated to Capital Medical University. Animal protocols were approved by the Ethics Committee of Beijing Chaoyang Hospital and performed in compliance with the institutional guidelines for the care and use of animals.

The AR (Lewis to BN) of OLT was performed according to Kamada's 2-cuff method. The donor livers were harvested from 8-10-week-old Lewis rats following ligation of the inferior vena cava, portal vein, and superior vena cava. The superior vena cava was then excised and attached through end-to-end anastomoses to the inferior vena cava of a recipient $\mathrm{BN}$ rat. The inferior vena cava and portal vein were anastomosed using the classic 2-cuff technique, and the hepatic artery was not constructed.

\section{Construction of the AR model of rat OLT with overexpression of FGL2}

pAAV-CMV-MCS-3FLAG-P2A-mNeonGreen-CW3SL [adeno-associated virus (AAV)-null] and pAAV-CMVFgl23Flag-P2A-mNeongreen-CW3SL (AAV-FGL2) were purchased from OBiO Technology (Shanghai, China). Lewis (donor) rats received an equal dose of AAV-FGL2 and AAV-null viral genome $\left(3 \times 10^{12}\right.$ vector genomes $\left./ \mathrm{kg}\right)$ before OLT (Lewis to $\mathrm{BN}$ ) via the tail vein. The following controls for the experimental groups of animals were included: blank group ( $\mathrm{n}=18$ ), an AR transplant group (Lewis to $\mathrm{BN}$ ) that did not receive any treatments; FGL2+ group $(\mathrm{n}=24)$, an AR transplant group (Lewis to $\mathrm{BN}$ ) that received donor with FGL2 intervention; and control group $(n=18)$, an $\mathrm{AR}$ transplant group (Lewis to $\mathrm{BN}$ ) that received donor with empty vector AAV intervention. The recipients did not receive any preoperative or postoperative treatments. The RNA levels and protein levels of FGL2 in the liver and spleen were detected by quantitative polymerase chain reaction (qPCR) and western blotting, respectively.

\section{Flow cytometry analysis}

Cells from peripheral blood, liver, and spleen were harvested to analyze immune cell phenotypes using flow cytometry. Target cells were freshly isolated from the spleens and livers of rats. Rats were killed by cervical dislocation, and spleen and liver were removed and crushed to separate target cells by grinding with a syringe plunger against a nylon net. 
The cells were filtered through $100-\mu \mathrm{m}$ and $70-\mu \mathrm{m}$ mesh strainers (Becton, Dickinson and Co. Bioscience, San Jose, CA, USA). Then, the cells were centrifuged at $1,500 \mathrm{rpm}$ for $5 \mathrm{~min}$. A total of $1 \mathrm{~mL}$ red blood cell lysate was used to remove red blood cells, which were then incubated at room temperature and kept away from light for $15 \mathrm{~min}$, washed with phosphate buffered saline (PBS), and centrifuged again under the same conditions.

A total of $200 \mu \mathrm{L}$ of peripheral blood were used for flow cytometry to detect the content of CD8+CD45RClow Tregs. The target cells were stained with APC-labelled CD8 antibody (BD Biosciences) and phycoerythrin (PE)-labelled CD45RC antibody (OX-22-Biotin, BioLegend, San Diego, CA, USA) at room temperature for $10 \mathrm{~min}$. Red blood cells were lysed with fluorescence-activated cell sorting (FACS) lysing solution, incubated at room temperature for $20 \mathrm{~min}$, and protected from light. A BD Bioscience FACS Aria II flow cytometer was used for cell acquisition, and Flowjo ${ }^{\mathrm{TM}}$ (V10) software was used for data analysis (https://www. flowjo.com/).

\section{Quantitative reverse transcription polymerase chain reaction}

The total RNA of recipient livers and spleens were isolated using Qiagen RNA Minikit (Tiangen Biotech, Beijing, China). The RNA quantity and quality were detected using the ND-1000 Spectrophotometer (NanodropTM, Rockland, DE, USA). The first-strand complement DNA (cDNA) was synthesized by a Quantscript Reverse Transcriptase Kit (Tiangen Biotech, Beijing, China). The quantitative reverse transcription polymerase chain reaction qRT-PCR was performed by using SYBR green qPCR mix (Tiangen Biotech, Beijing, China) according to manufacturer's instructions. The optimal qRT-PCR efficacy was achieved using a cycling profile including a denaturation at $95^{\circ} \mathrm{C}$ for $15 \mathrm{~min}$, followed by 40 cycles of $10 \mathrm{~s}$ at $95^{\circ} \mathrm{C}, 20 \mathrm{~s}$ at $60{ }^{\circ} \mathrm{C}$, and $30 \mathrm{~s}$ at $72{ }^{\circ} \mathrm{C}$. Antiglyceraldehyde 3-phosphate dehydrogenase (GAPDH) antibody was used as the internal reference. The qRT-PCR test was executed in a fluorescence ration PCR instrument (LightCycler ${ }^{\circledR} 96$ System, Roche Life Science, Penzberg, Germany). All qRT-PCR experiments were performed in duplicate. The sequence of primer pairs for rat FGL2 was 5'-ATGGGAGCACCAACTTCACC-3' (forward) and 5'-TCGTACACGGCGTAAAGTGT-3' (reverse), GAPDH was 5'-GGCAAGTTCAACGGCACAG-3' (forward) and 5'-CGCCAGTAGACTCCACGACAT-3' (reverse).

\section{Western blotting}

Total protein was extracted from liver and spleen using radioimmunoprecipitation assay (RIPA) lysate containing a phosphate inhibitor (100:1 V/V) and protease inhibitor cocktail (100:1 V/V) following the standard procedure. Protein concentrations were detected using a bicinchoninic acid (BCA) protein quantitative kit (Beyotime, Jiangsu, China). A total of $30 \mu \mathrm{g}$ of total protein was added to $12 \%$ sodium dodecyl sulfate-polyacrylamide gel electrophoresis (SDS-PAGE) and then transferred to polyvinylidene fluoride (PVDF) membranes (Bio-Rad, Hercules, CA, USA). Primary antibodies against FGL2 (1:500) were used in western blotting following the manufacturer's instructions, with GAPDH $(1: 1,000)$ being used as a control. Primary antibodies were incubated in blocking solution overnight at $4{ }^{\circ} \mathrm{C}$. The next day, the membranes were incubated with horseradish peroxidase (HRP)-marked antibodies $(1: 2,000)$ at room temperature for $1 \mathrm{~h}$. Signals were detected through the chemiluminescent reaction using a gel imaging system (Bio-Rad).

\section{Hematoxylin and eosin (HE) staining}

HE staining was performed to assess the severity of rejection. The transplanted liver grafts were collected and fixed in $4 \%$ paraformaldehyde and embedded in paraffin, then sliced into $4-\mu \mathrm{m}$ thick sections and mounted onto glass slides, dried, and baked at $60^{\circ} \mathrm{C}$ for $30 \mathrm{~min}$ before the start of experimentation. The HE staining was performed following standard procedures. The results were observed under light microscopy.

\section{Immunohistochemistry (IHC)}

Allografts were processed for IHC. The preparation before conducting experiments was performed in the same way as that for samples used for HE staining. For immunoperoxidase staining, primary antibodies against FGL2 (rabbit anti-FGL2, 1:200) and CD8 (rabbit antiCD8, 1:200) were used at $4{ }^{\circ} \mathrm{C}$ overnight. The unbound antibody was removed with $1 \times$ tris-buffered saline with Tween 20 (TBST), and the sample was incubated with secondary antibodies for $2 \mathrm{~h}$ at room temperature with constant shaking. Incubations were separated by washing steps $(3 \times 3 \mathrm{~min}$ in TBST). The chromogen used was 
Table 1 Expression levels of CD8+CD45RClow Tregs according to different organs and times

\begin{tabular}{|c|c|c|c|c|c|c|c|c|c|}
\hline Group & \multicolumn{3}{|c|}{ Peripheral blood } & \multicolumn{3}{|c|}{ Liver } & \multicolumn{3}{|c|}{ Spleen } \\
\hline Blank group & $8.65 \pm 0.49$ & $13.06 \pm 0.72$ & - & $22.93 \pm 1.30$ & $16.06 \pm 0.66$ & - & $12.06 \pm 0.55$ & $2.90 \pm 0.64$ & - \\
\hline Control group & $8.60 \pm 0.14$ & $14.84 \pm 0.58$ & - & $23.7 \pm 1.15$ & $16.86 \pm 0.32$ & - & $10.43 \pm 0.66$ & $2.80 \pm 0.31$ & - \\
\hline FGL2+ group & $8.32 \pm 0.12^{*}$ & $47.15 \pm 4.95^{\star}$ & $8.36 \pm 0.19$ & $38.20 \pm 1.60$ & $44.80 \pm 1.30^{\star}$ & $52.33 \pm 1.90$ & $11.83 \pm 0.75$ & $2.84 \pm 0.25$ & $2.65 \pm 0.33$ \\
\hline
\end{tabular}

${ }^{*} \mathrm{P}<0.05$ when compared with the blank group. $\mathrm{CD}$, cluster of differentiation; FGL2, fibrinogen-like protein 2; Tregs, regulatory $T$ cells.

3,3-diaminobenzidine (DAB). The results were rated by 3 independent researchers blinded to the results.

\section{Statistical analysis}

SPSS 22.0 (IBM, Armonk, NY, USA) software was used for statistical analysis. Data are expressed as mean $\pm \mathrm{SD}$. The Student's t-test was used to analyze 2 samples, and one-way analysis of variance (ANOVA) was used for different groups. The Kaplan-Meier method was used to analyze overall survival (OS). A $\mathrm{P}$ value $<0.05$ was considered statistically significant.

\section{Results}

\section{Tolerogenic CD8+ Treg accumulated in the allograft}

First, we explored the effect on the Tregs in the AR model of liver transplantation. The presence of CD8+DC45RClow T cells was analyzed in the peripheral blood, allograft, and spleen on the 7th, 14th, and 30th postoperative days after liver transplantation (Table 1). As demonstrated in Figure 1A,B, treatment with AAV-FGL2 increased CD8+CD45RClow Tregs subsets in the liver of OLT rats compared with blank groups (POD 7, POD 14). Furthermore, the proportion of CD8+CD45RClow Tregs in the allografts was gradually increased with the prolongation of survival time. In the peripheral blood, the percentage of CD8+CD45RClow Treg cells on the 7th postoperative day was not discernibly different between different groups, whereas on the 14th postoperative day, increased numbers of CD8+CD45RClow Treg cells were observed in the FGL2+ groups compared with blank and control groups (Figure 1C,D). In the spleen, there was no significant difference in the expression level of CD8+CD45RClow Tregs across the 3 groups, but the expression level of CD8+CD45RClow Tregs showed a significant decrease with the lengthening of survival time (Figure 1E,F).
Thus, long-term allograft survival was associated with the accumulation of CD8+CD45RClow Tregs in the allograft.

\section{High expression of FGL2 appeared in the early acute stages}

As shown in Figure 2, Lewis rats were transfected with AAV-expressing FGL2 (AAV-FGL2) and green fluorescent protein, GFP (AAV-null). The expression level of FGL2 in the liver and spleen was confirmed by qRT-PCR and western blotting with virus transfection being used as a control on the 28th day after administration. Western blotting was used to examine the expression level of FGL2 in the liver and spleen following viral AAV injection. The results revealed that FGL2 was expressed at a high level in the liver but not the spleen, and the difference was statistically significant (Figure 2A,B). The qRT-PCR results showed that FGL2 expression in the liver was upregulated in the FGL2+ group compared with the control group, whereas no changes were observed in the expression of FGL2 in the spleen (Figure 2C).

The qRT-PCR results between the control and FGL2+ groups revealed that the expression level of FGL2 was not appreciably different, indicating that the AAV did not affect FGL2 expression (Figure 2C). As demonstrated in Figure 2C and Table 2, the qRT-PCR results of the liver and spleen showed that the expression level of FGL2 was highest on the 7 th postoperative day and then began to decrease on the 14th and 30th postoperative days in the FGL2+ group. Contrastingly, the expression was increased in the control group. The OS time of all the rats in the control group was less than or equal to 14 days, so observation of this group was not noted until 14 days. We used a western blotting assay to validate FGL2 expression levels in different AR groups and different organs. We observed significantly higher FGL2 expression in the FGL2+ group as compared to the blank group in the liver on the 7 th postoperative day; on the contrary, higher FGL2 expression was observed 
A

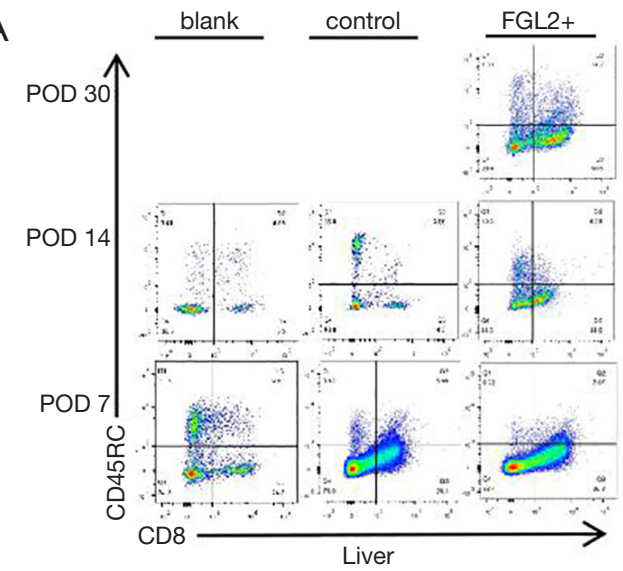

C

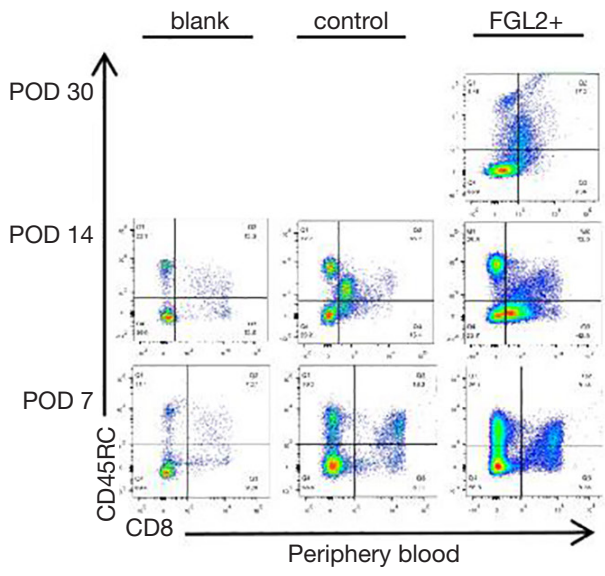

$\mathrm{E}$

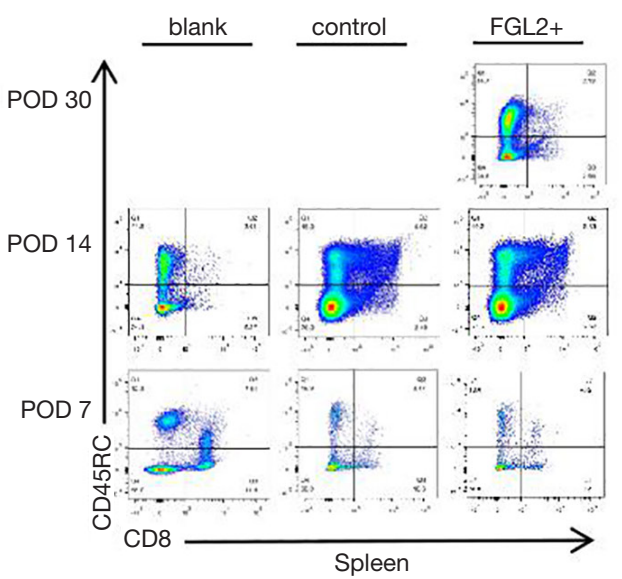

B

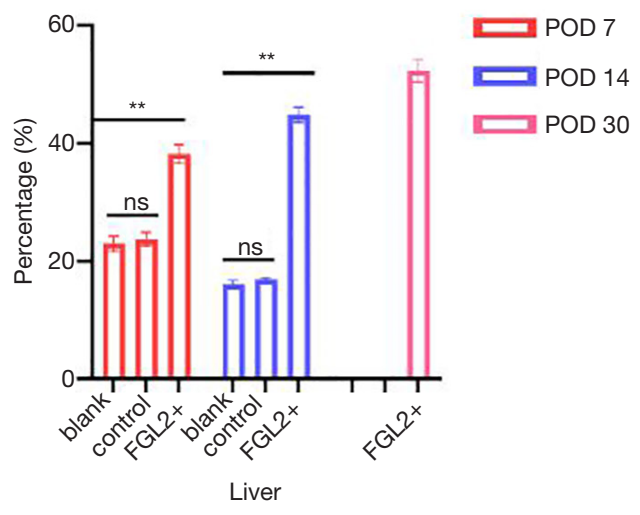

D

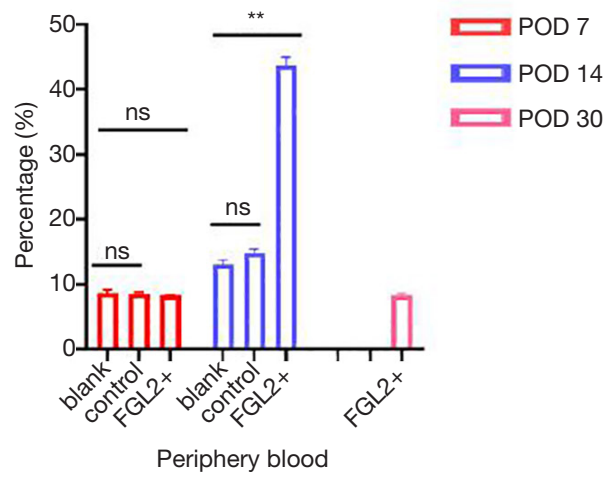

$\mathrm{F}$

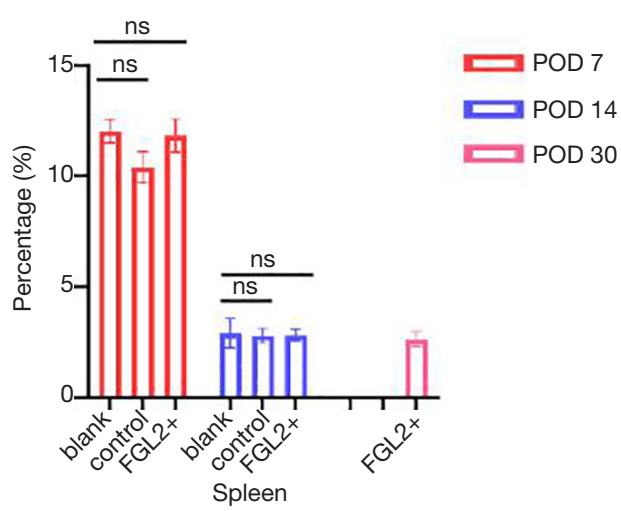

Figure 1 CD8+CD45RClow Tregs accumulated in allografts. Allografts, periphery blood, and the spleen of blank, control, and FGL2+ rats were analyzed for CD8+CD45RClow Treg populations on days 7,14, and 30 post-liver transplantation. $(\mathrm{A}, \mathrm{B})$ The expression levels of CD8+CD45RClow Tregs were detected by flow cytometry in allografts from rats of the blank, control, and FGL2+ groups. (C,D) Flow cytometry plots of CD8+CD45RClow Tregs from periphery blood of recipients. (E,F) CD8+CD45RClow Tregs expression levels were detected in the spleen of recipients. Data $(\mathrm{n}=6)$ are presented as mean \pm SD. One-way ANOVA was used to calculate the 2-sided $\mathrm{P}$ values. Graphs are representative of 6 independent experiments. ${ }^{* *} \mathrm{P}<0.01$, significant results. $\mathrm{P}>0.05$ indicates non-significance. $\mathrm{CD}$, cluster of differentiation; FGL2, fibrinogen-like protein 2; Tregs, regulatory T cells; SD, standard deviation; ANOVA, analysis of variance. 
A

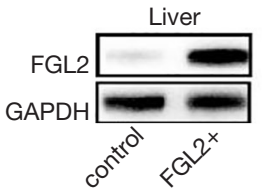

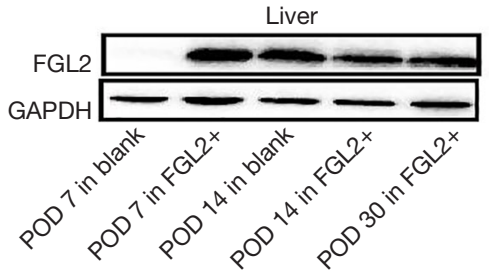
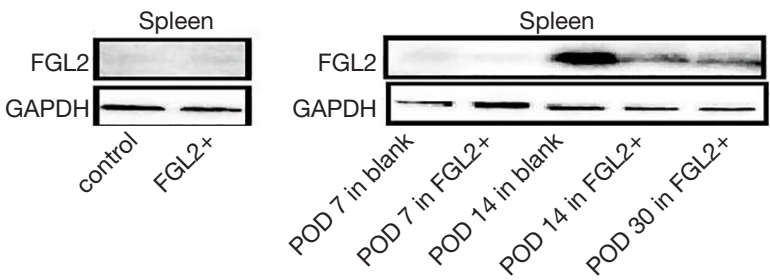

B
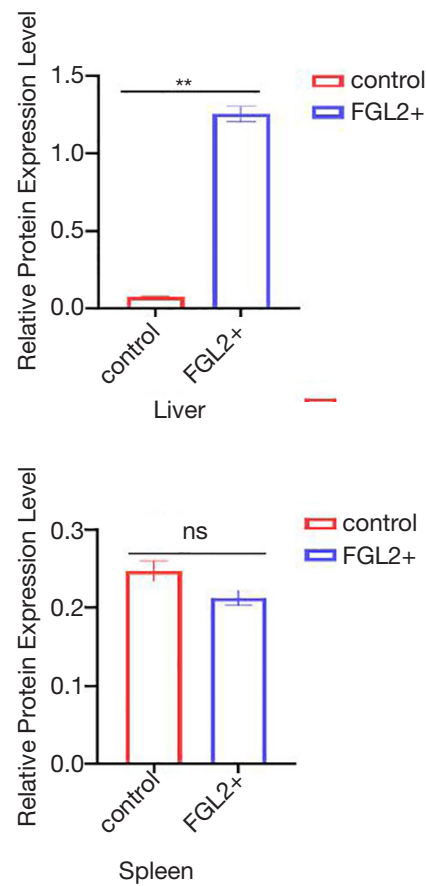
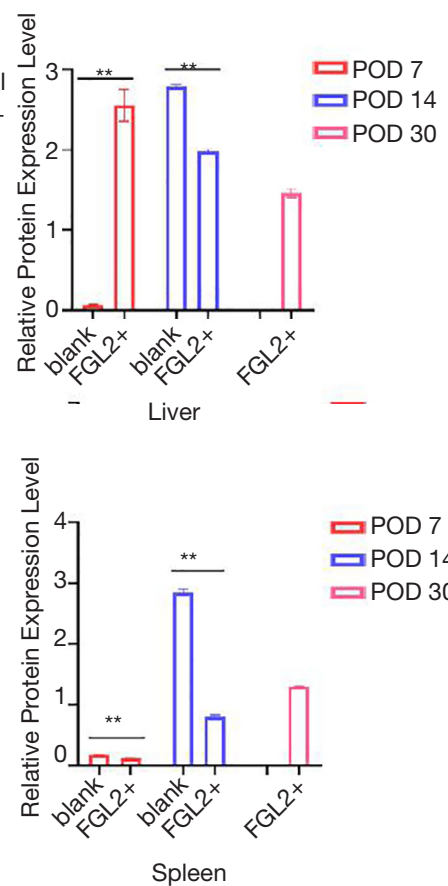
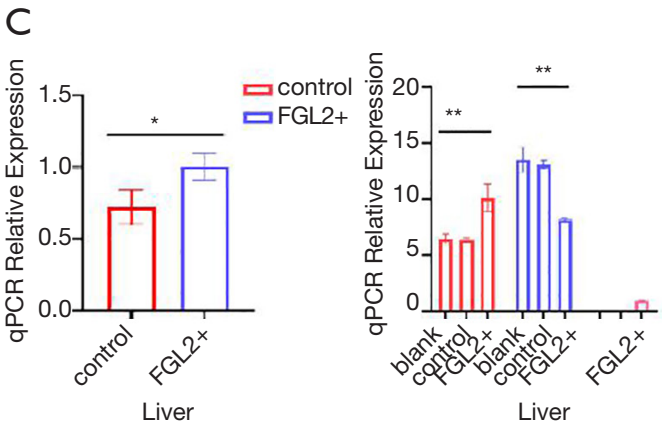

DPOD 7 口POD 14 口POD 30

Figure 2 Increased FGL2 in the FGL2+ group after rat liver transplantation. (A-C) The protein levels and mRNA of FGL2 in liver and spleen tissues from Lewis rats were examined using qRT-PCR or western blotting. (A,B) The protein levels of FGL2 in the blank and FGL2+ group were measured using western blotting. (C) The mRNA levels of FGL2 in liver and spleen tissues from BN rats and those in the allograft and spleen from recipients in the blank or FGL2+ group were examined by qRT-PCR. Graphs are representative of 6 independent experiments. Significant results are presented as ${ }^{*} \mathrm{P}<0.05,{ }^{* *} \mathrm{P}<0.01$. $\mathrm{P}>0.05$ indicates non-significance. FGL2, fibrinogen-like protein 2 ; mRNA, messenger ribonucleic acid; qRT-PCR, quantitative reverse transcription polymerase chain reaction; BN, Brown Norwegian.

Table 2 The RNA levels of FGL2 in liver and spleen according to different time points

\begin{tabular}{|c|c|c|c|c|c|c|}
\hline Group & \multicolumn{3}{|c|}{ Liver } & \multicolumn{3}{|c|}{ Spleen } \\
\hline Blank group & $6.50 \pm 0.42$ & $13.53 \pm 1.10$ & - & $0.84 \pm 0.08$ & $1.66 \pm 0.17$ & - \\
\hline Control group & $6.35 \pm 0.17$ & $12.09 \pm 0.39$ & - & $1.00 \pm 0.05$ & $1.70 \pm 0.05$ & - \\
\hline FGL2+ group & $10.12 \pm 1.24^{*}$ & $8.15 \pm 0.17^{\star}$ & $1.00 \pm 0.02$ & $2.74 \pm 0.01^{*}$ & $0.38 \pm 0.01^{\star}$ & $0.79 \pm 0.01$ \\
\hline
\end{tabular}

${ }^{*} \mathrm{P}<0.05$ when compared with blank group. FGL2, fibrinogen-like protein 2. 
Table 3 The protein levels of FGL2 in liver and spleen according to different time points

\begin{tabular}{|c|c|c|c|c|c|c|}
\hline Group & \multicolumn{3}{|c|}{ Liver } & \multicolumn{3}{|c|}{ Spleen } \\
\hline Blank group & $0.07 \pm 0.01$ & $2.79 \pm 0.02$ & - & $0.18 \pm 0.01$ & $2.85 \pm 0.05$ & - \\
\hline FGL2+ group & $2.56 \pm 0.20^{\star}$ & $1.97 \pm 0.03^{*}$ & $1.46 \pm 0.06$ & $0.12 \pm 0.01^{*}$ & $0.81 \pm 0.02^{*}$ & $1.30 \pm 0.01$ \\
\hline
\end{tabular}

${ }^{*} \mathrm{P}<0.05$ when compared with blank group. FGL2, fibrinogen-like protein 2.

A
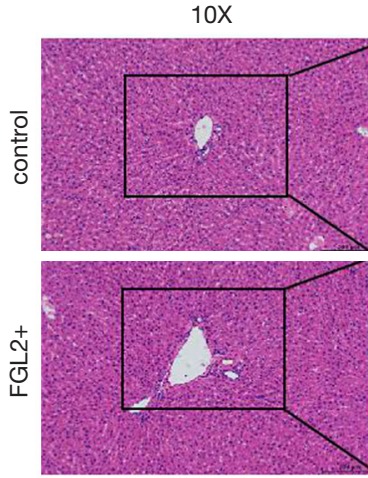

C
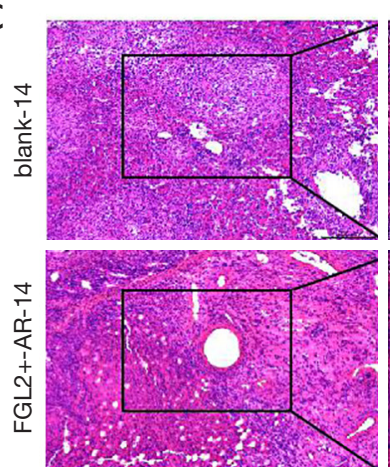

20X
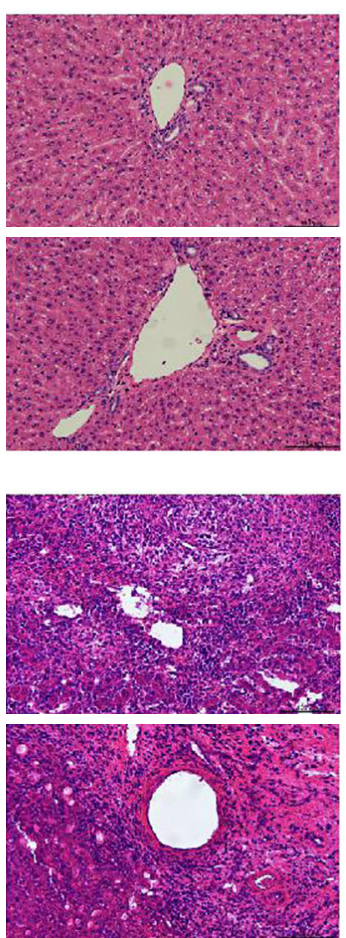

B

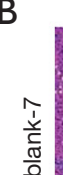
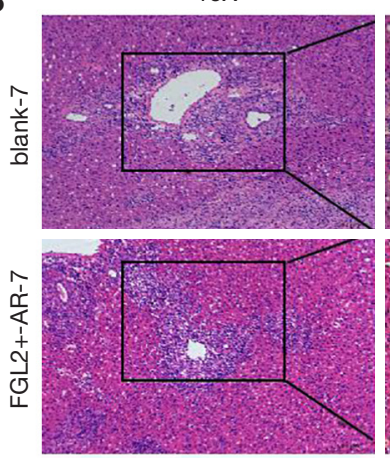

D

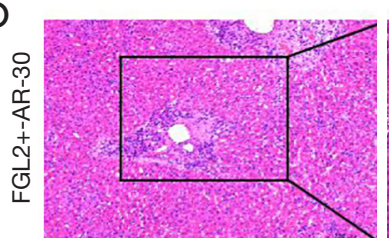

$20 X$
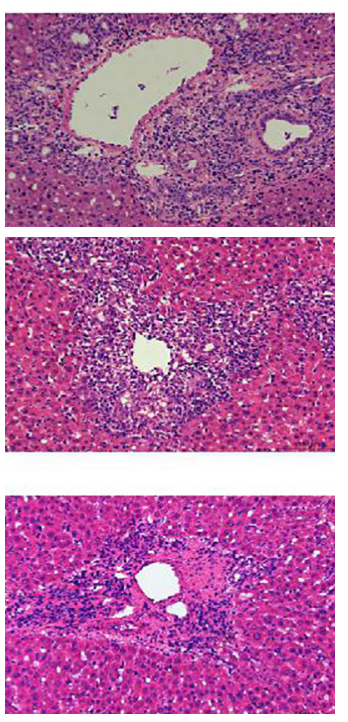

Figure 3 Infection with AAV-FGL2 led to the alleviation of acute rejection in the model of AR liver transplantation. (A) Livers were isolated from non-transplanted rats at 28 days postinfection with $3 \times 10^{12}$ vector genomes $/ \mathrm{kg}$. HE staining (magnification $10 \times, 20 \times$ ) revealed that nontransplanted livers did not show any signs of rejection or inflammation. (B,C) Significant inflammatory cell infiltration was observed in the allografts at days 7 and 14 postoperation; as compared with the blank group, the allografts from the FGL2+ group showed a lesser degree of acute rejection. (D) Inflammatory cells were significantly decreased in the allografts. The images and the survival curve represent 6 rats per group. AAV, adeno-associated virus; FGL2, fibrinogen-like protein 2; AR, acute rejection: HE, hematoxylin and eosin.

in the blank group on the 14 th postoperative day. In the FGL2+ group, FGL2 expression reached its peak on day 7 . In the spleen, the expression of FGL2 in the FGL2+ group was higher compared with that in the blank group on the 7th and 14th postoperative day. In the experimental groups, the expression level of FGL2 increased with a longer survival time (Table 3). These results suggested that OS correlated with increased FGL2 expression.

\section{High expression of FGL2 alleviated the AR}

To examine whether FGL2 might alleviate AR, liver transplantation was performed for 28 days after AAV infection. The results of HE staining revealed that the virus did not affect tissues of rats treated with AAV-null and without viral injection (Figure $3 A$ ). The HE staining examination for allografts showed less mononuclear cell 
A
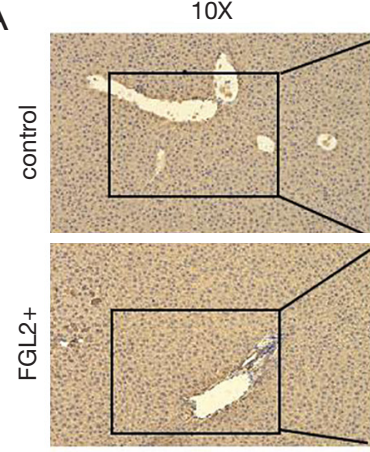

C

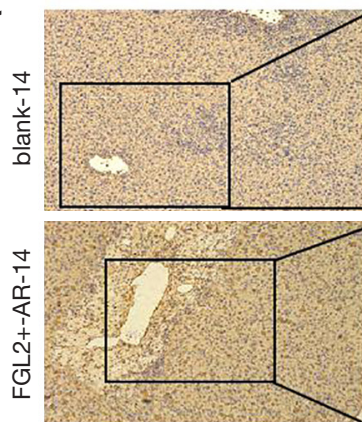

20X
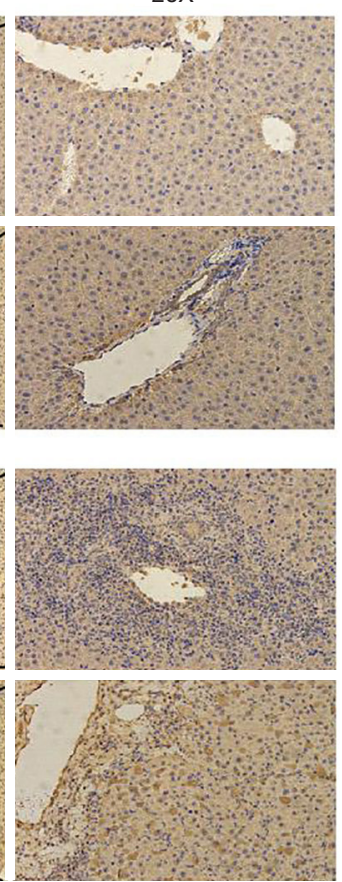

B
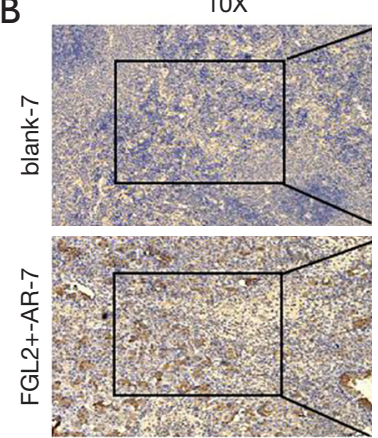

D

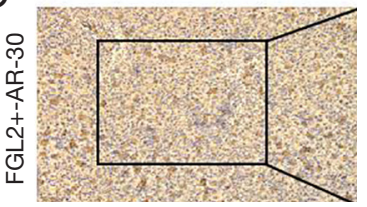

$20 X$
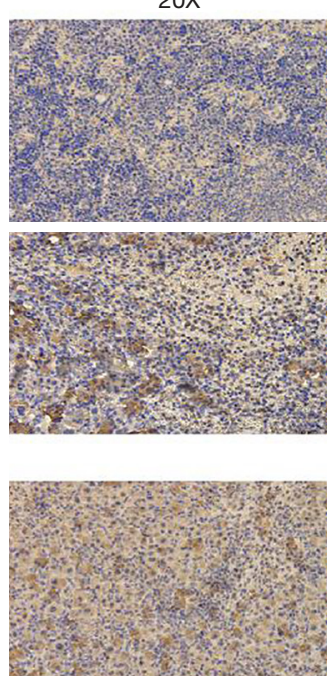

Figure 4 FGL2 levels increased with survival time. FGL2 IHC staining (magnification 10×, 20x) in nontransplanted livers (A), POD 7 allografts (B), POD 14 allografts (C), and POD 30 allografts (D). IHC staining (magnification 10×, 20x) revealed that nontransplanted livers did not show any signs of FGL2. (B,C) Significantly increased infiltration of FGL2 was observed in the allografts at days 7 and 14 postoperation, as compared with the blank group. (D) Significantly increased FGL2 levels were observed in the allografts at day 30 postoperation, as compared with the blank group. The images and survival curves represent 6 rats per group. IHC, immunohistochemical; FGL2, fibrinogen-like protein 2; POD, postoperative day.

(lymphocytes, neutrophils, and eosinophils) infiltration in the portal tracts, less venous endothelial inflammation, and milder bile duct damage in the FGL2+ groups compared with the blank groups. The results revealed no noticeable differences in the allografts of the FGL2+ group compared with the blank group on the 7th postoperative day (Figure 3B). However, on the 14th day, the blank group underwent severe rejection, characterized by massive of lymphocyte infiltration, and rejection occurred in the liver grafts (Figure $3 C$ ). On day 30, the severity of AR in the FGL2+ group was significantly reduced, and lymphocyte infiltration was markedly decreased (Figure 3D), indicating that FGL2 treatment could significantly alleviate AR following liver transplantation.

\section{CD8 + T cells decreased while FGL2 increased in grafts with the prolongation of survival time}

To investigate whether FGL2 can alleviate AR in vivo, we next assessed CD8+ T cells and FGL2 in the allograft by IHC. Indeed, the expression level of FGL2 increased alongside survival time (Figure $4 A, B, C, D$ ). The analysis could not be performed on allografts from control groups on the 30th day because these rats had already died. The OS of recipients of FGL2+ donors was significantly longer than that of the blank and control groups. Moreover, longer surviving rats in the FGL2+ group exhibited an increase in the proportion of FGL2 and a decrease of CD8+ T cells as compared with rats in both blank and control groups. There was no significant difference in CD8 expression compared with the control group (Figure $5 A$ ). On day 7 , in comparison with the FGL2+ group, large numbers of CD8+ $\mathrm{T}$ cells were observed in allografts of the blank group with dense mononuclear cell infiltration present (Figure 5B). On the 14th day, the results followed the same trend as that of the 7 th day (Figure 5C). In grafts studied 30 days post-transplantation, few if any CD8+ cells were found (Figure 5D). In contrast, levels of FGL2 were found to be elevated in allografts from FGL2+ groups on postoperative days 7,14 , and 30 . Moreover, the median 
A

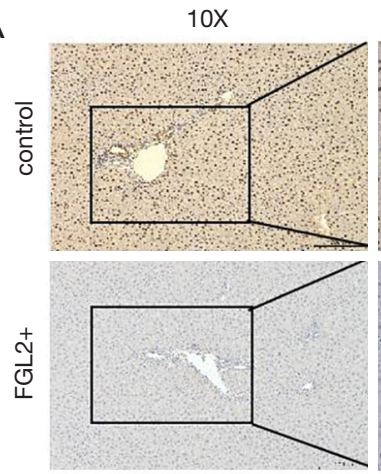

C

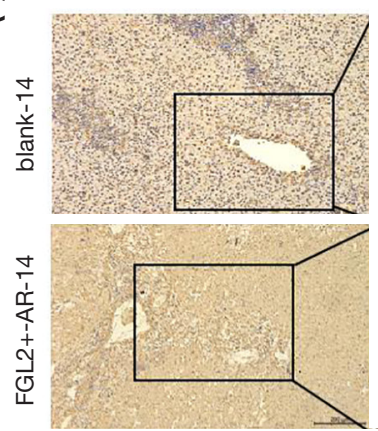

$20 X$
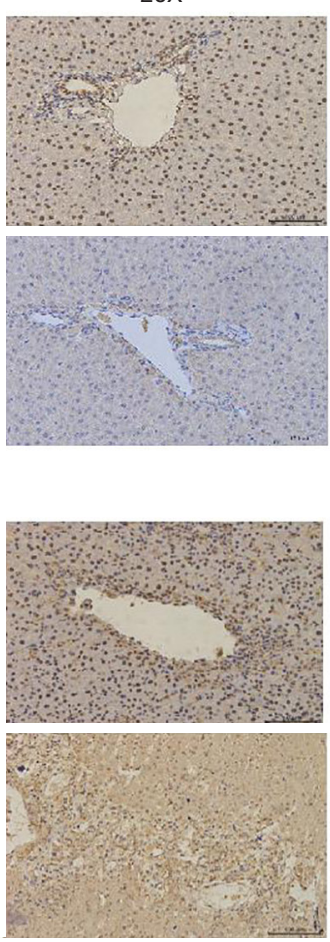

B
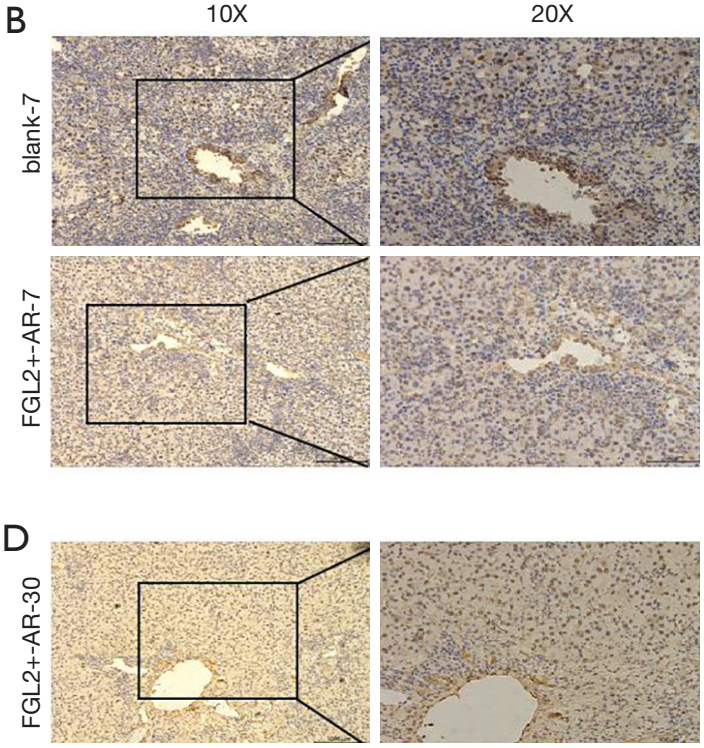

Figure 5 CD8 IHC staining (magnification 10x, 20x) in nontransplanted livers (A), POD 7 allografts (B), POD 14 allografts (C), and POD 30 allografts (D). A. IHC staining (magnification 10x, 20x) revealed that nontransplanted livers did not show any signs of CD8. (B,C) Significantly decreased CD8 infiltrating the allografts on POD 7 and POD 14, compared with the blank group. (D) Significantly decreased CD8 levels observed in the allografts at day 30 postoperation. The images and survival curves represent 6 rats per group. CD, cluster of differentiation; POD, postoperative day; IHC, immunohistochemical.

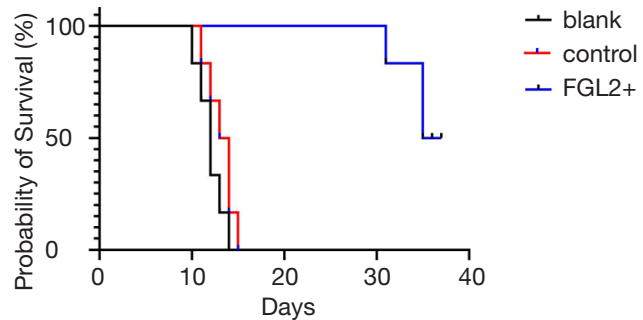

Figure 6 FGL2 treatment prolonged the survival of rats' liver transplantation allografts. Survival of Lewis livers transplanted into $\mathrm{BN}$ recipient rats. Recipient groups included a blank group (mean survival time $=12$ days, $\mathrm{n}=6$ ), control groups (median survival time $=13.5$ days, $\mathrm{n}=6$ ), and FGL2 + groups (mean survival time $=36$ days, $\mathrm{n}=6$ ). FGL2, fibrinogen-like protein 2; BN, Brown Norwegian.

survival time was 35.5 days (31-37 days) in the FGL2 treatment group, 12 days (10-14 days) in the blank group, and 13.5 days (11-15 days) in the control group (Figure 6). The results above show a stronger association between
FGL2 and alleviation of AR.

\section{Discussion}

Using a OLT model of AR in rats, we examined the tissue distribution and phenotypic changes underlying alleviation rejection by CD8+CD45RClow Tregs following overexpression FGL2 intervention. We first confirmed the accumulation of CD8+CD45RClow Tregs in allografts of rats transfected by AAV-FGL2 after liver transplantation and found that FGL2 levels increased with survival time. We constructed an AR model with AAV transfection to improve the prognosis of liver transplantation. It was found that treatment with AAV-FGL2 was able to attenuate AR and significantly prolong the survival of recipients.

As one of the most extensively studied T cells, Tregs are essential immune-suppressing cells, with the most widely studied being CD4+CD25+forkhead box protein (FOXP3) Tregs $(6,19)$. In addition to CD4+ Tregs, CD8+ 
Tregs, including Foxp3+, CD122+, or CD8 $\alpha \alpha+$, might also play a vital role in inhibiting immune reaction (20,22-24). In specific organs such as the eye, CD8+ Tregs likely play a predominant role in maintaining immune tolerance. A previous study reported that tolerogenic $\mathrm{CD} 8+\mathrm{CD} 45 \mathrm{RClow}$ Tregs, which generate infectious tolerance upon adoptive transfer, were induced by blockade of CD40-CD40L interactions with CD40Ig (25). Our previous study reported that tolerance induction was associated with an accumulation of CD8+CD45RClow Tregs in the graft and the spleen (11). In addition, CD8+ Tregs have been reported to accumulate in allografts (26), tumor sites (27), and inflamed lymphoid tissue (28). In this study, marked increases in the levels of CD8+CD45RClow Tregs in the peripheral blood of rats during the early period of $\mathrm{AR}$ were observed and at a later time-point, the levels of CD8+CD45RClow Tregs returned to baseline in the FGL2+ group.

Interestingly, the highest levels of FGL2 were observed during AR, which coincided with a large influx of $\mathrm{T}$ cells into the graft (1). Hence, the findings suggest that the levels of CD8+CD45RClow Tregs in peripheral blood may play an essential role in the early period of AR, which is consistent with evidence of severe cell-mediated rejection and so could be an attempt by the host to alleviate graft injury. Our study revealed that the expression level of FGL2 in the liver was higher in the AR group transfected by FGL2+ compared with those in the blank and control groups. Importantly, we found that the accumulation of CD8+CD45RClow Tregs first occurs within the allografts. The percentage exhibited a continuous increment in the allograft with prolonged survival time. This result is in line with the local immune tolerance in the liver.

Tregs are known to mediate graft acceptance through many mechanisms, including generating inhibitory cytokines, induction of apoptosis in effector cells, and inhibition of DC function. The FGL2 molecule is a newly found Treg-effector inhibitory molecule with immunosuppressive properties. As a crucial Treg effector molecule, FGL2 may exert effects of tolerance induction. Studies have reported that FGL2 could play essential roles in hepatic and cardiac transplantation tolerance, with overexpression of FGL2 being found to contribute to the tolerance of a mismatched heart allograft in mice $(14,29)$. Our study showed that mRNA and protein levels of FGL2 were higher in the livers of the FGL2+ group than those in the blank and control groups. Furthermore, compared with allografts of both the blank and control groups, the allografts of overexpressed FGL2 were associated with lower numbers of $\mathrm{CD} 8+\mathrm{T}$ cells and higher numbers FGL2+ molecules. Our previous study showed that the FGL2 molecule is a critical Treg effector in a rat model of transplant tolerance induced by costimulation blockade and is necessary for contact-dependent inhibition of effector T cells by CD8+ Treg (11). These results support our contention that FGL2 may be the critically important molecule for AR. The increase of FGL2 in allografts may represent a host response to control inflammation within the allograft. Analyses of the above results indicated that FGL2 might be the key molecule for maintaining transplant tolerance. These results align with the study reported by Gao et al. In this study, sFGL2-mesenchymal stem cells (sFGL2-MSCs) could inhibit AR after heart transplantation in mice by regulating macrophage activation. Meanwhile, Pan et al. (30) demonstrated that sFGL2 could induce the M2 polarization of Kupffer cells by suppressing the STAT1 and nuclear factor kappa-light-chain-enhancer of activated $\mathrm{B}$ cells (NF-KB) signaling pathways.

In conclusion, we have demonstrated that CD8+CD45RClow Tregs and FGL2 may exert effects for AR in a rat liver allograft model. The finding that CD8+CD45RClow Tregs had accumulated in the allograft suggests that FGL2induced alleviation of rejection may involve active immune suppression by CD8+CD45RClow Tregs. Future studies are needed to determine if molecular therapies based on FGL2 can alleviate rejection and further achieve tolerance in the setting of liver transplantation. It is possible that FGL2 may act as a tissue-specific immune inhibitory molecule. Tissuespecific knockdown of FGL2 is necessary to determine if FGL2 may exert an effect for rejection alleviation and tolerance induction.

\section{Acknowledgments}

We extend our thanks to Drs. Ren Lang, Hua Fan, Jiantao Kou, Yu Liu, and Lixin Li for their general support.

Funding: This work was supported by the Chaoyang 1351 Talent Training Plan (Year 2020).

\section{Footnote}

Reporting Checklist: The authors have completed the ARRIVE reporting Checklist. Available at http://dx.doi. org/10.21037/atm-20-7881

Data Sharing Statement: Available at http://dx.doi. 
org/10.21037/atm-20-7881

Peer Review File: Available at http://dx.doi.org/10.21037/ atm-20-7881

Conflicts of Interest: All authors have completed the ICMJE uniform disclosure form (available at http://dx.doi. org/10.21037/atm-20-7881). The authors have no conflicts of interest to declare.

Ethical Statement: The authors are accountable for all aspects of the work in ensuring that questions related to the accuracy or integrity of any part of the work are appropriately investigated and resolved. This study was performed under a project license (no. 2019-D.-102) granted by the Ethics Committee of Beijing Chaoyang Hospital and was performed in compliance with the institutional guidelines for the care and use of animals.

Open Access Statement: This is an Open Access article distributed in accordance with the Creative Commons Attribution-NonCommercial-NoDerivs 4.0 International License (CC BY-NC-ND 4.0), which permits the noncommercial replication and distribution of the article with the strict proviso that no changes or edits are made and the original work is properly cited (including links to both the formal publication through the relevant DOI and the license). See: https://creativecommons.org/licenses/by-nc-nd/4.0/.

\section{References}

1. Sykes M, Levy G. Advances in transplantation. Semin Immunol 2011;23:222-3.

2. Knechtle SJ, Kwun J. Unique aspects of rejection and tolerance in liver transplantation. Semin Liver Dis 2009;29:91-101.

3. Goldfarb SB, Benden C, Edwards LB, et al. The Registry of the International Society for Heart and Lung Transplantation: Eighteenth Official Pediatric Lung and Heart-Lung Transplantation Report--2015; Focus Theme: Early Graft Failure. J Heart Lung Transplant 2015;34:1255-63.

4. Kaufman DB, Shapiro R, Lucey MR, et al. Immunosuppression: practice and trends. Am J Transplant 2004;4 Suppl 9:38-53.

5. Pallardó Mateu LM, Sancho Calabuig A, Capdevila Plaza $\mathrm{L}$, et al. Acute rejection and late renal transplant failure: risk factors and prognosis. Nephrol Dial Transplant
2004;19 Suppl 3:iii38-42.

6. San Segundo D, Fernandez-Fresnedo G, Ruiz JC, et al. Two-year follow-up of a prospective study of circulating regulatory $\mathrm{T}$ cells in renal transplant patients. Clin Transplant 2010;24:386-93.

7. Wood KJ, Sakaguchi S. Regulatory T cells in transplantation tolerance. Nat Rev Immunol 2003;3:199-210.

8. Wood KJ, Bushell A, Hester J. Regulatory immune cells in transplantation. Nat Rev Immunol 2012;12:417-30.

9. Gondek DC, Lu LF, Quezada SA, et al. Cutting edge: contact-mediated suppression by CD4+CD25+ regulatory cells involves a granzyme B-dependent, perforinindependent mechanism. J Immunol 2005;174:1783-6.

10. Pandiyan $\mathrm{P}$, Zheng $\mathrm{L}$, Ishihara $\mathrm{S}$, et al. $\mathrm{CD} 4+\mathrm{CD} 25+$ Foxp3 + regulatory $\mathrm{T}$ cells induce cytokine deprivation-mediated apoptosis of effector $\mathrm{CD} 4+\mathrm{T}$ cells. Nat Immunol 2007;8:1353-62.

11. Li XL, Menoret S, Bezie S, et al. Mechanism and localization of $\mathrm{CD} 8$ regulatory $\mathrm{T}$ cells in a heart transplant model of tolerance. J Immunol 2010;185:823-33.

12. Shevach EM. Mechanisms of foxp3 + T regulatory cellmediated suppression. Immunity 2009;30:636-45.

13. Shalev I, Liu H, Koscik C, et al. Targeted deletion of fgl2 leads to impaired regulatory $T$ cell activity and development of autoimmune glomerulonephritis. J Immunol 2008;180:249-60.

14. Bartczak A, Chruscinski A, Mendicino M, et al. Overexpression of Fibrinogen-Like Protein 2 Promotes Tolerance in a Fully Mismatched Murine Model of Heart Transplantation. Am J Transplant 2016;16:1739-50.

15. Urbanellis $\mathrm{P}$, Shyu $\mathrm{W}$, Khattar R, et al. The regulatory $\mathrm{T}$ cell effector molecule fibrinogen-like protein 2 is necessary for the development of rapamycin-induced tolerance to fully MHC-mismatched murine cardiac allografts. Immunology 2015;144:91-106.

16. Morris AB, Farley CR, Pinelli DF, et al. Signaling through the Inhibitory Fc Receptor FcgammaRIIB Induces CD8(+) T Cell Apoptosis to Limit T Cell Immunity. Immunity 2020;52:136-50.e6.

17. Liu H, Shalev I, Manuel J, et al. The FGL2FcgammaRIIB pathway: a novel mechanism leading to immunosuppression. Eur J Immunol 2008;38:3114-26.

18. Chan CW, Kay LS, Khadaroo RG, et al. Soluble fibrinogen-like protein 2/fibroleukin exhibits immunosuppressive properties: suppressing $\mathrm{T}$ cell proliferation and inhibiting maturation of bone marrowderived dendritic cells. J Immunol 2003;170:4036-44. 
19. Graca L, Thompson S, Lin CY, et al. Both CD4(+) CD25(+) and CD4(+)CD25(-) regulatory cells mediate dominant transplantation tolerance. J Immunol 2002;168:5558-65.

20. Vinay DS, Kim CH, Choi BK, et al. Origins and functional basis of regulatory CD11c+CD8+ T cells. Eur J Immunol 2009;39:1552-63.

21. Shalev I, Wong KM, Foerster K, et al. The novel CD4+CD25+ regulatory $T$ cell effector molecule fibrinogen-like protein 2 contributes to the outcome of murine fulminant viral hepatitis. Hepatology 2009;49:387-97.

22. Tang X, Maricic I, Purohit N, et al. Regulation of immunity by a novel population of Qa-1-restricted CD8alphaalpha+TCRalphabeta+ T cells. J Immunol 2006; 177:7645-55.

23. Xystrakis E, Dejean AS, Bernard I, et al. Identification of a novel natural regulatory CD8 T-cell subset and analysis of its mechanism of regulation. Blood 2004;104:3294-301.

24. Endharti AT, Rifa IM, Shi Z, et al. Cutting edge: CD8+CD122+ regulatory $\mathrm{T}$ cells produce IL-10 to suppress IFN-gamma production and proliferation of CD8+ T cells. J Immunol 2005;175:7093-7.

Cite this article as: Zhang $\mathrm{X}, \mathrm{Ma} \mathrm{J}, \mathrm{Li} \mathrm{H}$, Zhou L, Liu Z, Lyu S, He Q, Li X. Overexpression of fibrinogen-like protein 2 alleviates acute rejection in rat models of liver transplantation. Ann Transl Med 2021;9(5):408. doi: 10.21037/atm-20-7881
25. Guillonneau C, Hill M, Hubert FX, et al. CD40Ig treatment results in allograft acceptance mediated by CD8CD45RC T cells, IFN-gamma, and indoleamine 2,3-dioxygenase. J Clin Invest 2007;117:1096-106.

26. Izawa A, Yamaura K, Albin MJ, et al. A novel alloantigenspecific CD8+PD1+ regulatory T cell induced by ICOSB7h blockade in vivo. J Immunol 2007;179:786-96.

27. Wei S, Kryczek I, Zou L, et al. Plasmacytoid dendritic cells induce CD8+ regulatory $\mathrm{T}$ cells in human ovarian carcinoma. Cancer Res 2005;65:5020-6.

28. Siegmund K, Ruckert B, Ouaked N, et al. Unique phenotype of human tonsillar and in vitro-induced FOXP3+CD8+ T cells. J Immunol 2009;182:2124-30.

29. Xie L, Ichimaru N, Morita M, et al. Identification of a novel biomarker gene set with sensitivity and specificity for distinguishing between allograft rejection and tolerance. Liver Transpl 2012;18:444-54.

30. Pan G, Zhao Z, Tang C, et al. Soluble fibrinogenlike protein 2 ameliorates acute rejection of liver transplantation in rat via inducing Kupffer cells M2 polarization. Cancer Med 2018;7:3168-77.

(English Language Editors: J. Jones and J. Gray) 